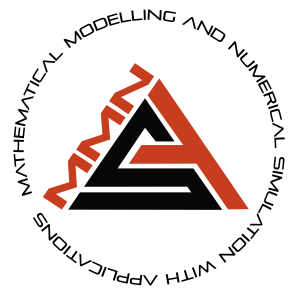

\title{
A numerical approach to the coupled atmospheric ocean model using a fractional operator
}

\author{
Pundikala Veeresha $1, *, \dagger$ \\ ${ }^{1}$ Center for Mathematical Needs, Department of Mathematics, CHRIST (Deemed to be University), \\ Bengaluru-560029, India \\ *Corresponding Author \\ †pundikala.veeresha@christuniversity.in \& pveeresha.maths@gmail.com
}

\begin{abstract}
In the present framework, the coupled mathematical model of the atmosphere-ocean system called El Nino-Southern Oscillation (ENSO) is analyzed with the aid Adams-Bashforth numerical scheme. The fundamental aim of the present work is to demonstrate the chaotic behaviour of the coupled fractional-order system. The existence and uniqueness are demonstrated within the frame of the fixed-point hypothesis with the Caputo-Fabrizio fractional operator. Moreover, we captured the chaotic behaviour for the attained results with diverse order. The effect of the perturbation parameter and others associated with the model is captured. The obtained results elucidate that, the present study helps to understand the importance of fractional order and also initial conditions for the nonlinear models to analyze and capture the corresponding consequence of the fractional-order dynamical systems.
\end{abstract}

Key words: Caputo-Fabrizio derivative; El Nino-Southern oscillation model; fixed point theorem AMS 2020 Classification: 34A08; 26A33; 65L05

\section{Introduction}

The study of mathematical models is always a venue for innovation. It attracted researchers to illustrate their viewpoints and forecast the future significances of the associated phenomena. In this regard, the most efficient and reliable tool is calculus with both integral and differential operators. Most of the phenomena associated with the rate of change are modelled to assist these operators in the modernization of day-day life. For instance, the security of the country, biological processes, economic status, physical mechanism, chemical reaction, weather forecast, coastal and ocean engineering, and many others are examined as well moderated with the aid of mathematical modelling. Moreover, it becomes an interdisciplinary subject due to its ability to exemplify complex phenomena, and also it plays a vital role in creating a bridge between diverse areas. However, many researchers proved that the generalization of classical calculus is very essential to capture the more complex nature of the nonlinear problems associated with daily life. Later, they suggest the concept of calculus with fractional order called fractional calculus (FC) $[1,2,3,4,5]$. Even though it originated earlier, it recently fascinated scholars to investigate more essential behaviours the mathematical models described by differential equations $[6,7,8,9,10]$.

On the other hand, the study of climate with irregularly intervallic changes in sea surface and wind temperatures is a hot topic in the present era due to its significance in diverse fields associated with living beings. Here, we consider the mathematical model exemplifying the atmospheric component coupled with the sea temperature change high air surface pressure, called El Nino-Southern Oscillation (ENSO) [11, 12, 13, 14]. In the tropical western Pacific, La Niña is with low air surface pressure and El Niño with high air surface pressure. The dynamics of recount the oscillating physical mechanism of the ENSO model with the

> Received: 24.06.2021 > Revised: 11.08.2021 > Accepted: 13.08.2021 > Published: 15.08.2021 
thermocline depth anomaly $v(t)$ and temperature of the eastern equatorial Pacific Sea surface $u(t)$ is presented with perturbation coefficient $\varepsilon$ as $[15,16,17,18]$ follows

$$
\begin{aligned}
& \frac{d u(t)}{d t}=\beta u+\eta v-\varepsilon u^{3}, \\
& \frac{d v(t)}{d t}=-\theta u-\gamma v,
\end{aligned}
$$

where $\beta, \eta, \theta$ and $\gamma$ are physical constants. The projected coupled system plays an important role in various phenomena. The projected system is analysed by many researchers to present their viewpoints and also capture linear and complex nature using many semi-analytical and numerical schemes $[11,12,13,14,15,16,17,18]$. In the literature, we have distinct fractional operators each one has its own limitation while examining the complex phenomena. In the present investigation, we consider the fractional operator without singular kernel, called Caputo-Fabrizio (CF) operator in Caputo sense [6], to examine the projected system. Due to more ability to capture the complex nature associated with history-based consequences and memory-related properties, FC is an interdisciplinary subject. Its fundamentals and theories are considered to study diverse real-world problems and attain numerous essential results [19, 20, 21, 22, 23, 24, 25, 26, 27, 28, 29]. Notably, results associated with hereditary, memory, random walk, longrange memory, non-Markovian processes, anomalous diffusion, and others highly necessitate the concept of FC. In this regard, authors in [36], the effect of generalizing the classical concept with the newly defined fractional operator to investigate about the HBV infection with antibody immune response, the nature of Belousov-Zhabotinskii reaction systems have been captured within the frame of Atangana-Baleanu fractional-order derivative by researchers in [37], some ingesting results are derived by authors in [38] about the vector born disease with the help of efficient scheme and Caputo-Fabrizio derivative, the strong interacting internal waves model has been examined with the reliable numerical method with a novel fractional operator in [39], and authors in $[40,41,42,43,44,45]$ derived some essential properties of the fractional operators.

Here, we consider Eq. (1) with the CF operator as follows

$$
\begin{aligned}
& { }_{0}^{C F} D_{t}^{\mu} u(t)=\beta u+\eta v-\varepsilon u^{3}, \\
& { }_{0}^{C F} D_{t}^{\mu} v(t)=-\theta u-\gamma v,
\end{aligned}
$$

where ${ }_{0}^{C F} D_{t}^{\mu}$ is a CF derivative with order $\mu$.

The rest of the investigation is organized as follows: we recalled basic definitions of the considered fractional operator in the next section. In Section 3, the basic algorithm of the considered method is presented, and in the next section, the condition for the existence and uniqueness of solutions for the projected system is illustrated. The results and discussion on the derived results are illustrated in Section 5, and concluding remarks are presented in the lost section.

\section{Preliminaries}

The basic notions of FC are recalled in the present segment [30, 31].

A real function $f(t), t>0$ is said to be in space $C_{v}, v \in \mathbb{R}$ if there exists a real number $k(>v)$, such that $f(t)=t^{k} f_{1}$, where $f_{1}(t) \in C[0, \infty)$, which is also in space if and only if $u^{(n)} \in C_{v}, n \in \mathbb{N}$.

Definition 1. The Caputo fractional derivative of $f \in C_{-1}^{n}$ is presented as

$$
D_{t}^{\mu} f(t)=\frac{1}{\Gamma(n-\mu)} \int_{0}^{t}(t-\vartheta)^{n-\mu-1} \frac{d^{n}}{d t^{n}} f(\vartheta) d \vartheta
$$

Definition 2. The Caputo-Fabrizio (CF) fractional derivative in Caputo sense for a function $f \in H^{1}(a, b)(b>a)$ is [6]

$$
{ }_{0}^{C F} D_{0, t}^{\mu}(f(t))=\frac{\mathcal{N}[\mu]}{1-\mu} \int_{0}^{t} f^{\prime}(\vartheta) \exp \left[-\frac{\mu(t-\vartheta)}{1-\mu}\right] d \vartheta
$$

where $\mathcal{N}[\mu](\mathcal{N}[0]=\mathcal{N}[1]=1)$ is normalization function

But, in case the function $u$ does not belong to $H^{1}(a, b)$, the CF derivative for this version is defined as

$$
{ }_{0}^{C F} D_{0, t}^{\mu}(f(t))=\frac{\mu \mathcal{N}[\mu]}{1-\mu} \int_{0}^{t} f(t)-f(\vartheta) \exp \left[-\frac{\mu(t-\vartheta)}{1-\mu}\right] d \vartheta .
$$

Later, Losada and Nieto in [20] modified the above CF fractional derivative as follows

$$
{ }_{0}^{C F} D_{0, t}^{\mu}(f(t))=\frac{(2-\mu) \mathcal{N}[\mu]}{2(1-\mu)} \int_{0}^{t} f^{\prime}(\vartheta) \exp \left[-\frac{\mu(t-\vartheta)}{1-\mu}\right] d \vartheta
$$

Definition 3. The Caputo-Fabrizio (CF) fractional integral for $\mu \in(0,1)$ is defined as

$$
{ }_{0}^{C F} I_{t}^{\mu}(f(t))=\frac{1-\mu}{\mathcal{N}[\mu]} f(t)+\frac{\mu}{\mathcal{N}[\mu]} \int_{0}^{t} f(\vartheta) d \vartheta, t \geq 0 .
$$




\section{Numerical method}

In this segment, with the Caputo-Fabrizio operator, the two-step Adams-Bashforth method is hired [32, 33, 34]

$$
{ }_{0}^{C F} D_{t}^{\mu}(u(t))=\frac{\mathcal{N}[\mu]}{1-\mu} \int_{0}^{t} u^{\prime}(\vartheta) \exp \left[-\mu \frac{t-\vartheta}{1-\mu}\right] d \vartheta .
$$

Now, the grid size for some integer $N$ for finite difference method is $k=\frac{1}{N}$. Further, the grid points are presented in the time interval $[0, T]$ as $t_{n}=n k, n=0,1,2, \ldots, T N$. At the grid point, the value of the function $u_{i}=u\left(t_{i}\right)$. For the fractional-order Caputo-Fabrizio derivative, a discrete approximation is presented as [34]

$$
{ }_{0}^{C F} D_{t}^{\mu}\left(u\left(t_{n}\right)\right)=\frac{\mathcal{N}[\mu]}{1-\mu} \int_{0}^{t_{n}} u^{\prime}(\vartheta) \exp \left[-\mu \frac{t_{n}-\vartheta}{1-\mu}\right] d \vartheta .
$$

By the assist of first-order approximation, the above equation simplifies

$$
{ }_{0}^{C F} D_{t}^{\mu}\left(u\left(t_{j}\right)\right)=\frac{\mathcal{N}[\mu]}{1-\mu} \sum_{j=1}^{n} \int_{(j-1) k}^{j k}\left(\frac{u^{k+1}-u^{k}}{\Delta t}+\mathcal{O}(\Delta t)\right) \exp \left[-\mu \frac{t_{j}-\vartheta}{1-\mu}\right] d \vartheta
$$

But

$$
\begin{gathered}
\frac{\mathcal{N}[\mu]}{1-\mu} \sum_{j=1}^{n}\left(\frac{u^{j+1}-u^{j}}{\Delta t}+\mathcal{O}(\Delta t)\right) \int_{(j-1) k}^{j k} \exp \left[-\mu \frac{t_{j}-\vartheta}{1-\mu}\right] d \vartheta, \\
{ }_{0}^{C F} D_{t}^{\mu}\left(u\left(t_{j}\right)\right)=\frac{\mathcal{N}[\mu]}{\mu} \sum_{j=1}^{n}\left(\frac{u^{j+1}-u^{j}}{\Delta t}+\mathcal{O}(\Delta t)\right) d_{j, k},
\end{gathered}
$$

where

$$
d_{j, k}=\exp \left[-\mu \frac{k}{1-\mu}(n-j+1)\right]-\exp \left[-\mu \frac{k}{1-\mu}(n-j)\right] .
$$

Finally, we obtained

$$
{ }_{0}^{C F} D_{t}^{\mu}\left(u\left(t_{n}\right)\right)=\frac{\mathcal{N}[\mu]}{\mu} \sum_{j=1}^{n}\left(\frac{u^{j+1}-u^{j}}{\Delta t}\right) d_{j, k}+\frac{\mathcal{N}[\mu]}{\mu} \sum_{j=1}^{n} d_{j, k} \mathcal{O}(\Delta t) .
$$

\section{Existence and uniqueness of solutions}

Here, present the existence and uniqueness of the hired model within the frame of the fixed-point theorem. The system defined in Eq. (2) hired as follows

$$
\left\{\begin{array}{c}
{ }_{0} F D_{t}^{\mu}[u(t)]=\mathcal{G}_{1}(t, u)=\beta u+\eta v-\varepsilon u^{3} \\
{ }_{0}^{C F} D_{t}^{\mu}[v(t)]=\mathcal{G}_{2}(t, v)=-\theta u-\gamma v
\end{array}\right.
$$

Now, using Eq. (2), we have

$$
\begin{aligned}
& u(t)-u(0)={ }_{0}^{C F} I_{t}^{\mu}\left\{\beta u+\eta v-\varepsilon u^{3}\right\}, \\
& v(t)-v(0)={ }_{0}^{C F} I_{t}^{\mu}\{-\theta u-\gamma v\} .
\end{aligned}
$$

Then we have from [35] as follows

$$
\begin{aligned}
& u(t)-u(0)=\frac{2 \mu}{(2-\mu) \mathcal{N}(\mu)} \int_{0}^{t} \mathcal{G}_{1}(\zeta, u) d \zeta+\frac{2(1-\mu)}{(2-\mu) \mathcal{N}(\mu)} \mathcal{G}_{1}(t, u), \\
& v(t)-v(0)=\frac{2 \mu}{(2-\mu) \mathcal{N}(\mu)} \int_{0}^{t} \mathcal{G}_{2}(\zeta, v) d \zeta+\frac{2(1-\mu)}{(2-\mu) \mathcal{N}(\mu)} \mathcal{G}_{2}(t, v) .
\end{aligned}
$$

Theorem 1. The kernels $\mathcal{G}_{1}$ and $\mathcal{G}_{2}$ satisfies the Lipschitz condition and contraction if $0 \leq\left(\beta+\eta \lambda_{2}-\varepsilon\left(a^{2}+b^{2}+a b\right)\right)<1$ and $0 \leq\left(\theta \lambda_{1}+\gamma\right)<1$, satisfies respectively. 
Proof. We consider the two functions $u$ and $u_{1}$ to prove the required result, then

$$
\begin{aligned}
\left\|\mathcal{G}_{1}(t, u)-\mathcal{G}_{1}\left(t, u_{1}\right)\right\| & =\left\|\left(\beta\left[u(t)-u\left(t_{1}\right)\right]+\eta v(t)-\varepsilon\left[u^{3}(t)-u^{3}\left(t_{1}\right)\right]\right)\right\| \\
& =\left\|\left(\beta\left[u(t)-u\left(t_{1}\right)\right]+\eta v(t)-\varepsilon\left[u^{2}(t)+u^{2}\left(t_{1}\right)+u(t) u\left(t_{1}\right)\right]\left[u(t)-u\left(t_{1}\right)\right]\right)\right\| \\
& \leq\left\|\beta+\eta v(t)-\varepsilon\left(a^{2}+b^{2}+a b\right)\right\|\left\|u(t)-u\left(t_{1}\right)\right\| \\
& \leq\left(\beta+\eta \lambda_{2}-\varepsilon\left(a^{2}+b^{2}+a b\right)\right)\left\|u(t)-u\left(t_{1}\right)\right\|,
\end{aligned}
$$

where $\|v(t)\| \leq \lambda_{2}$ be the bounded function. Since $u$ and $u_{1}$ are bounded, we have $\|u(t)\| \leq a$ and $\left\|u\left(t_{1}\right)\right\| \leq b$. Setting $\rho_{1}=$ $\beta+\eta \lambda_{2}-\varepsilon\left(a^{2}+b^{2}+a b\right)$ in the above inequality, then we have

$$
\left\|\mathcal{G}_{1}(t, u)-\mathcal{G}_{1}\left(t, u_{1}\right)\right\| \leq \rho_{1}\left\|u(t)-u\left(t_{1}\right)\right\| .
$$

Eq. (19) provides the Lipschitz condition for $\mathcal{G}_{1}$. Similarly, we can see that if $0 \leq\left(\beta+\eta \lambda_{2}-\varepsilon\left(a^{2}+b^{2}+a b\right)\right)<1$, then it implies the contraction. In the same way for $\rho_{2}=\theta \lambda_{1}+\gamma$, we can prove

$$
\left\|\mathcal{G}_{2}(t, v)-\mathcal{G}_{2}\left(t, v_{1}\right)\right\| \leq \rho_{2}\left\|v(t)-v\left(t_{1}\right)\right\|
$$

By the assist of Eq. (20), Eq. (17) gives

$$
\begin{aligned}
& u(t)=u(0)+\frac{2 \mu}{(2-\mu) \mathcal{N}(\mu)} \int_{0}^{t} \mathcal{G}_{1}(\zeta, u) d \zeta+\frac{2(1-\mu)}{(2-\mu) \mathcal{N}(\mu)} \mathcal{G}_{1}(t, u), \\
& v(t)=v(0)+\frac{2 \mu}{(2-\mu) \mathcal{N}(\mu)} \int_{0}^{t} \mathcal{G}_{2}(\zeta, v) d \zeta+\frac{2(1-\mu)}{(2-\mu) \mathcal{N}(\mu)} \mathcal{G}_{2}(t, v) .
\end{aligned}
$$

Then obtain the recursive form as

$$
\begin{aligned}
& u_{n}(t)=\frac{2 \mu}{(2-\mu) \mathcal{N}(\mu)} \int_{0}^{t} \mathcal{G}_{1}\left(\zeta, u_{n-1}\right) d \zeta+\frac{2(1-\mu)}{(2-\mu) \mathcal{N}(\mu)} \mathcal{G}_{1}\left(t, u_{n-1}\right), \\
& v_{n}(t)=\frac{2 \mu}{(2-\mu) \mathcal{N}(\mu)} \int_{0}^{t} \mathcal{G}_{1}\left(\zeta, v_{n-1}\right) d \zeta+\frac{2(1-\mu)}{(2-\mu) \mathcal{N}(\mu)} \mathcal{G}_{1}\left(t, v_{n-1}\right) .
\end{aligned}
$$

The associated initial conditions are

$$
u(0)=u_{0}(t) \text { and } v(0)=v_{0}(t) .
$$

Now, between the terms the successive difference is presented as

$$
\begin{aligned}
& \Phi_{1 n}(t)=u_{n}(t)-u_{n-1}(t)=\frac{2(1-\mu)}{(2-\mu) \mathcal{N}(\mu)}\left(\mathcal{G}_{1}\left(t, u_{n-1}\right)-\mathcal{G}_{1}\left(t, u_{n-2}\right)\right)+\frac{2 \mu}{(2-\mu) \mathcal{N}(\mu)} \int_{0}^{t}\left(\mathcal{G}_{1}\left(t, u_{n-1}\right)-\mathcal{G}_{1}\left(t, u_{n-2}\right)\right) d \zeta \\
& \Phi_{2 n}(t)=v_{n}(t)-v_{n-1}(t)=\frac{2(1-\mu)}{(2-\mu) \mathcal{N}(\mu)}\left(\mathcal{G}_{2}\left(t, v_{n-1}\right)-\mathcal{G}_{2}\left(t, v_{n-2}\right)\right)+\frac{2 \mu}{(2-\mu) \mathcal{N}(\mu)} \int_{0}^{t}\left(\mathcal{G}_{2}\left(t, v_{n-1}\right)-\mathcal{G}_{2}\left(t, v_{n-2}\right)\right) d \zeta .
\end{aligned}
$$

Notice that

$$
\begin{aligned}
& u_{n}(t)=\sum_{i=1}^{n} \phi_{1 i}(t), \\
& v_{n}(t)=\sum_{i=1}^{n} \phi_{2 i}(t) .
\end{aligned}
$$

Therefore

$$
\left\|\phi_{1 n}(t)\right\|=\left\|u_{n}(t)-u_{n-1}(t)\right\|=\left\|\frac{2(1-\mu)}{(2-\mu) \mathcal{N}(\mu)}\left(\mathcal{G}_{1}\left(t, u_{n-1}\right)-\mathcal{G}_{1}\left(t, u_{n-2}\right)\right)+\frac{2 \mu}{(2-\mu) \mathcal{N}(\mu)} \int_{0}^{t}\left(\mathcal{G}_{1}\left(t, u_{n-1}\right)-\mathcal{G}_{1}\left(t, u_{n-2}\right)\right) d \zeta\right\| .
$$

The above equation simplifies with the assist of the triangular inequality, as

$$
\left\|\phi_{1 n}(t)\right\|=\left\|u_{n}(t)-u_{n-1}(t)\right\|=\frac{2(1-\mu)}{(2-\mu) \mathcal{N}(\mu)}\left\|\left(\mathcal{G}_{1}\left(t, u_{n-1}\right)-\mathcal{G}_{1}\left(t, u_{n-2}\right)\right)\right\|+\frac{2 \mu}{(2-\mu) \mathcal{N}(\mu)}\left\|\int_{0}^{t}\left(\mathcal{G}_{1}\left(t, u_{n-1}\right)-\mathcal{G}_{1}\left(t, u_{n-2}\right)\right) d \zeta\right\| .
$$

Then we have for $u$ admitting the Lipschitz condition

$$
\left\|\phi_{1 n}(t)\right\|=\left\|u_{n}(t)-u_{n-1}(t)\right\| \leq \frac{2(1-\mu)}{(2-\mu) \mathcal{N}(\mu)} \rho_{1}\left\|\phi_{1(n-1)}(t)\right\|+\frac{2 \mu}{(2-\mu) \mathcal{N}(\mu)} \rho_{1} \int_{0}^{t}\left\|\phi_{1(n-1)}(t)\right\| d \zeta .
$$


Similarly, one can get

$$
\left\|\phi_{2 n}(t)\right\| \leq \frac{2 \mu}{(2-\mu) \mathcal{N}(\mu)} \rho_{2} \int_{0}^{t}\left\|\phi_{2(n-1)}(\zeta)\right\| d \zeta+\frac{2(1-\mu)}{(2-\mu) \mathcal{N}(\mu)} \rho_{2}\left\|\phi_{2(n-1)}(t)\right\| .
$$

Now, we state the following theorem with the assist of the above attained results:

Theorem 2. If we have specific $t_{0}$, then the solution for Eq. (2) will exist and be unique. Further, we have for $i=1,2$.

$$
\frac{2(1-\mu)}{(2-\mu) \mathcal{N}(\mu)} \rho_{i}+\frac{2 \mu}{(2-\mu) \mathcal{N}(\mu)} \rho_{i} t_{0}<1,
$$

Proof. Let $u(t)$ and $v(t)$ be the bounded functions admitting the Lipschitz condition. Then, we get by Eqs. (28) and (29)

$$
\begin{aligned}
& \left\|\phi_{1 i}(t)\right\| \leq\|u(0)\|\left[\frac{2 \mu}{(2-\mu) \mathcal{N}(\mu)} \rho_{1} t+\frac{2(1-\mu)}{(2-\mu) \mathcal{N}(\mu)} \rho_{1}\right]^{n}, \\
& \left\|\Phi_{2 i}(t)\right\| \leq\left\|v_{n}(0)\right\|\left[\frac{2 \mu}{(2-\mu) \mathcal{N}(\mu)} \rho_{2} t+\frac{2(1-\mu)}{(2-\mu) \mathcal{N}(\mu)} \rho_{2}\right]^{n} .
\end{aligned}
$$

Therefore, for the obtained solutions, continuity and existence are verified. Now, to prove the Eq. (30) is a solution for Eq. (2), we consider

$$
\begin{aligned}
& u(t)-u(0)=u_{n}(t)-\mathcal{K}_{1 n}(t) \\
& v(t)-v(0)=v_{n}(t)-\mathcal{K}_{2 n}(t)
\end{aligned}
$$

Let us consider

$$
\begin{array}{r}
\left\|\mathcal{K}_{1 n}(t)\right\|=\left\|\frac{2(1-\mu)}{(2-\mu) \mathcal{N}(\mu)}\left(\mathcal{G}_{1}(t, u)-\mathcal{G}_{1}\left(t, u_{n-1}\right)\right)+\frac{2 \mu}{(2-\mu) \mathcal{N}(\mu)} \int_{0}^{t}\left(\mathcal{G}_{1}(\zeta, u)-\mathcal{G}_{1}\left(\zeta, u_{n-1}\right)\right) d \zeta\right\| \\
\leq \frac{2(1-\mu)}{(2-\mu) \mathcal{N}(\mu)}\left\|\left(\mathcal{G}_{1}(t, u)-\mathcal{G}_{1}\left(t, u_{n-1}\right)\right)\right\|+\frac{2 \mu}{(2-\mu) \mathcal{N}(\mu)} \int_{0}^{t}\left\|\left(\mathcal{G}_{1}(\zeta, u)-\mathcal{G}_{1}\left(\zeta, u_{n-1}\right)\right)\right\| d \zeta \\
\quad \leq \frac{2(1-\mu)}{(2-\mu) \mathcal{N}(\mu)} \rho_{1}\left\|u-u_{n-1}\right\|+\frac{2 \mu}{(2-\mu) \mathcal{N}(\mu)} \rho_{1}\left\|u-u_{n-1}\right\| t .
\end{array}
$$

This process gives

$$
\left\|\mathcal{K}_{1 n}(t)\right\| \leq\left(\frac{2 \mu}{(2-\mu) \mathcal{N}(\mu)} t+\frac{2(1-\mu)}{(2-\mu) \mathcal{N}(\mu)}\right)^{n+1} \rho_{1}^{n+1} M
$$

Similarly, at $t_{0}$ we can obtain

$$
\left\|\mathcal{K}_{1 n}(t)\right\| \leq\left(\frac{2 \mu}{(2-\mu) \mathcal{N}(\mu)} t_{0}+\frac{2(1-\mu)}{(2-\mu) \mathcal{N}(\mu)}\right)^{n+1} \rho_{1}^{n+1} M
$$

As $n \rightarrow \infty$ and from Eq. (33), $\left\|\mathcal{K}_{1 n}(t)\right\| \rightarrow 0$. Similarly, we can verify for $\left\|\mathcal{K}_{2 n}(t)\right\|$. Next, for the solution of the projected model, we prove its uniqueness. Suppose $u^{*}(t)$ and $v^{*}(t)$, be the set of other solutions, then

$$
u(t)-u^{*}(t)=\frac{2(1-\mu)}{(2-\mu) \mathcal{N}(\mu)}\left(\mathcal{G}_{1}(t, u)-\mathcal{G}_{1}\left(t, u^{*}\right)\right)+\frac{2 \mu}{(2-\mu) \mathcal{N}(\mu)} \int_{0}^{t}\left(\mathcal{G}_{1}(\zeta, u)-\mathcal{G}_{1}\left(\zeta, u^{*}\right)\right) d \zeta .
$$

Now, employing the norm on the above equation, we get

$$
\begin{aligned}
\left\|u(t)-u^{*}(t)\right\|=\| \frac{2(1-\mu)}{(2-\mu) \mathcal{N}(\mu)} & \left(\mathcal{G}_{1}(t, u)-\mathcal{G}_{1}\left(t, u^{*}\right)\right)+\frac{2 \mu}{(2-\mu) \mathcal{N}(\mu)} \int_{0}^{t}\left(\mathcal{G}_{1}(\zeta, u)-\mathcal{G}_{1}\left(\zeta, u^{*}\right)\right) d \zeta \| \\
\leq & \frac{2 \mu}{(2-\mu) \mathcal{N}(\mu)} \rho_{1} t\left\|u(t)-u^{*}(t)\right\|+\frac{2(1-\mu)}{(2-\mu) \mathcal{N}(\mu)} \rho_{1}\left\|u(t)-u^{*}(t)\right\| .
\end{aligned}
$$

On solving

$$
\left\|u(t)-u^{*}(t)\right\|\left(1-\frac{2 \mu}{(2-\mu) \mathcal{N}(\mu)} \rho_{1} t-\frac{2(1-\mu)}{(2-\mu) \mathcal{N}(\mu)} \rho_{1}\right) \leq 0 .
$$

From Eq. (36), it is clear that $u(t)=u^{*}(t)$, if

$$
\left(1-\frac{2 \mu}{(2-\mu) \mathcal{N}(\mu)} \rho_{1} t-\frac{2(1-\mu)}{(2-\mu) \mathcal{N}(\mu)} \rho_{1}\right) \geq 0 .
$$

Hence, Eq. (37) proves our required proof. 


\section{Results and discussion}

The study of complex nature associated with real-world models always attracted young researchers to present their viewpoints and illustrated novel properties of the corresponding system. In this work, we hired coupled system exemplifying the atmospheric ocean, namely ENSO model using a novel fractional derivative. The considered coupled system is analysed with the initial conditions $u(0)=u_{0}(t)=1$ and $v(0)=v_{0}(t)=1$. The nature of hired model for different $\mu$ is captured in Figure 1. From these figures, we can observe that as order increase, the complex nature reduces, specifically for $\mu=0.85$ we can observe more cycles in the plots. For different values of the perturbation parameter, the response of the achieved results is captured and cited in Figure 2 . For $\eta=4$, we can evidence the more complex nature as compared to $\eta=2$. Similarly, we captured the nature for distinct $\theta$ and presented it in Figure 3. The present investigation confirms that the slight changes in the physical parameters associated to systems and furthers will help us investigate and predict the corresponding essential behaviour of the system. The fractional operator allows us to capture the more complex nature of the same system associated with time-based properties.
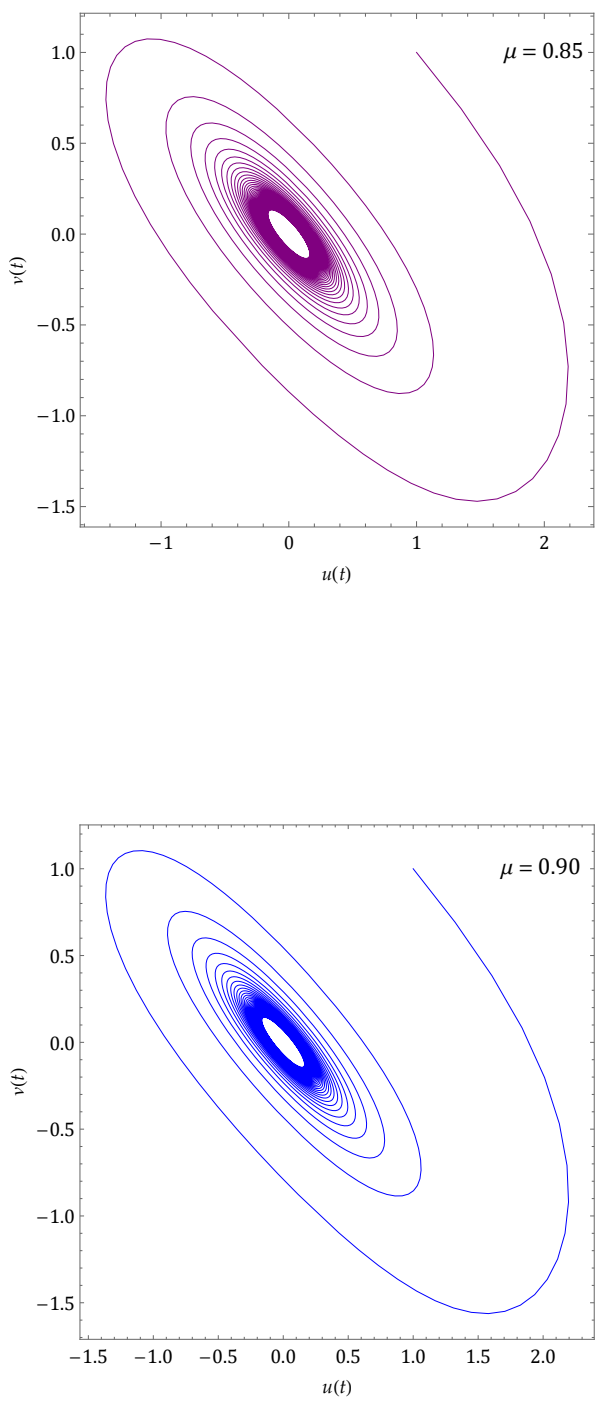

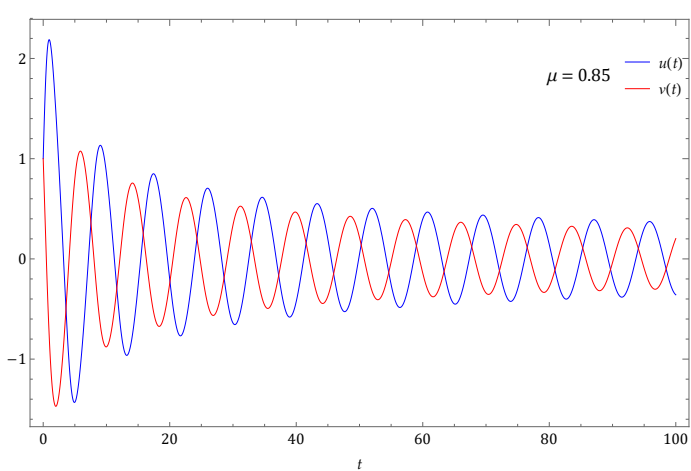

(a)

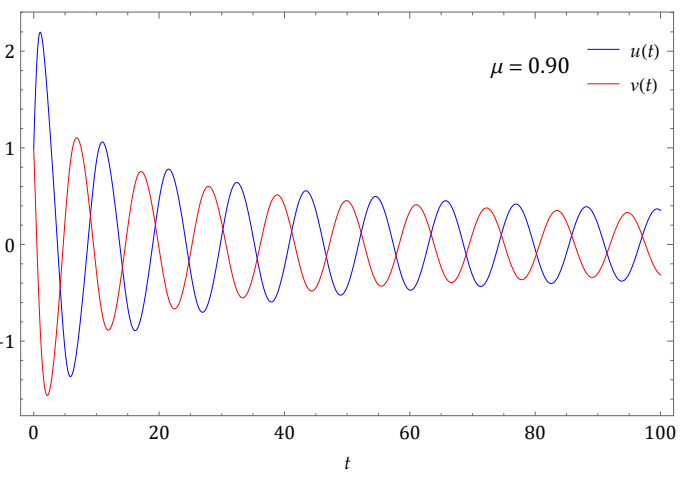

(b) 


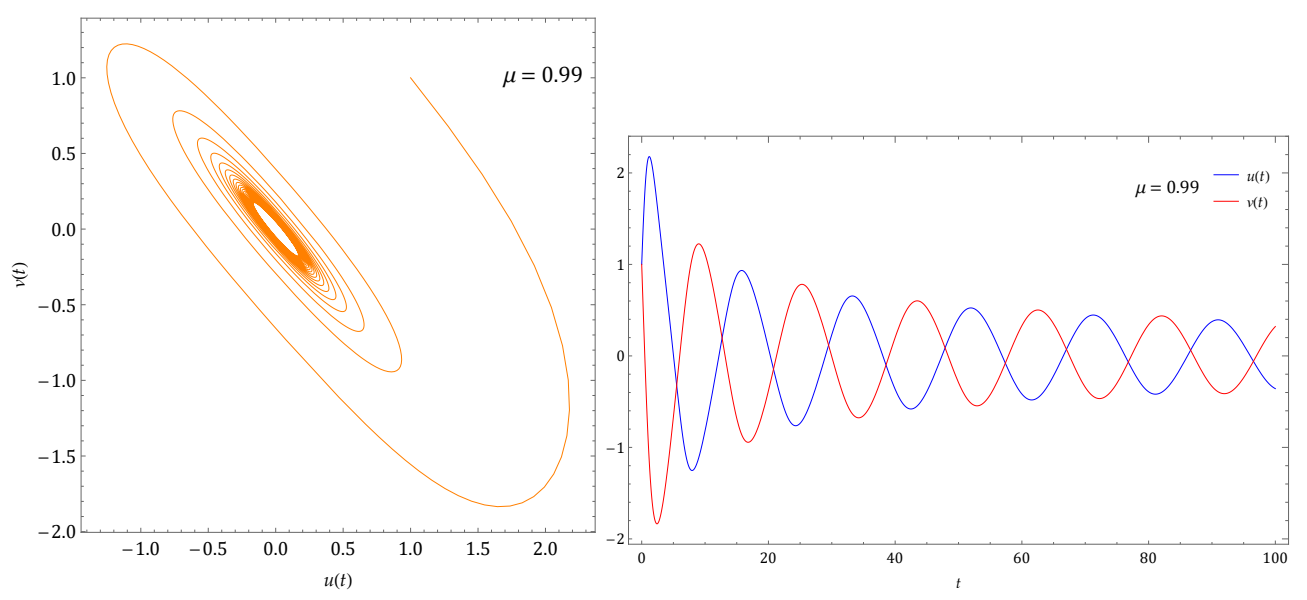

(c)

Figure 1. Behaviour of the results achieved for $(a) \mu=0.85,(b) \mu=0.90$ and $(c) \mu=0.99$ at $\beta=1, \eta=1, \theta=1, \gamma=1$ and $\epsilon=0.1$.
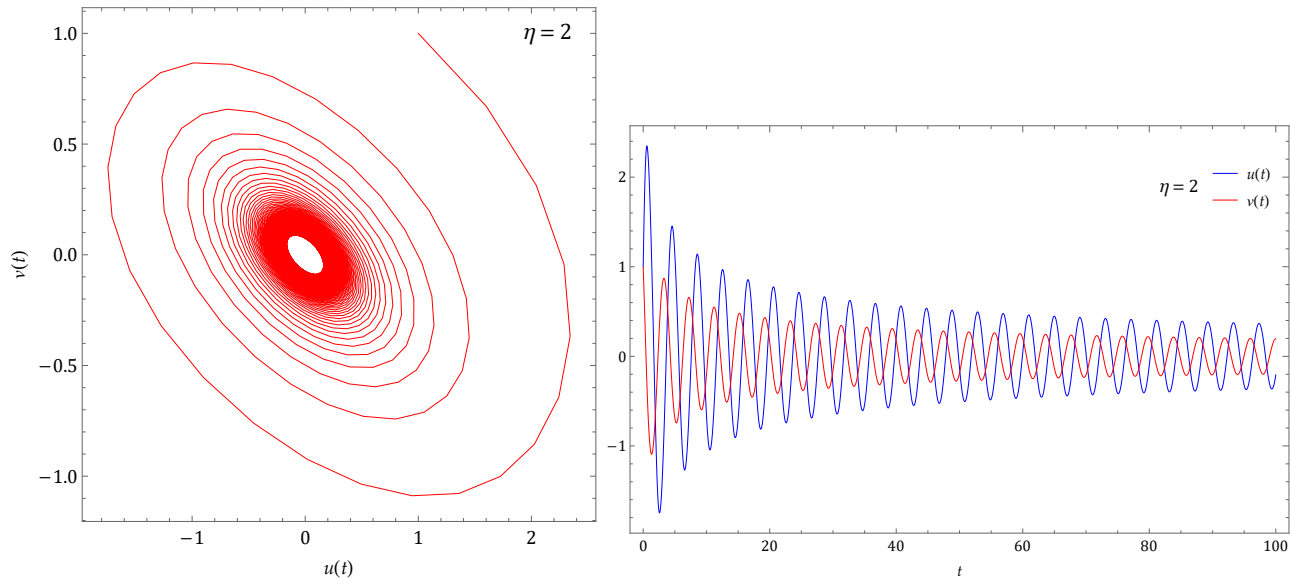

(a)
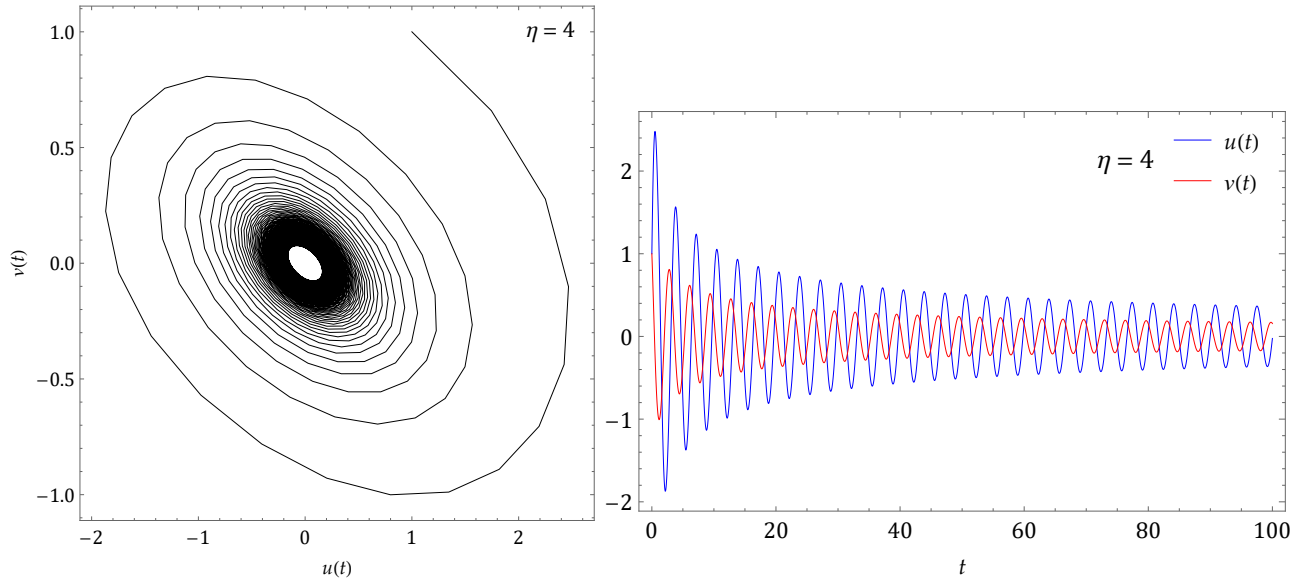

(b)

Figure 2. Nature of the results achieved for $(a) \eta=2$ and $(b) \mu=4$ at $\beta=1, \mu=0.95, \theta=1, \gamma=1$ and $\epsilon=0.1$. 

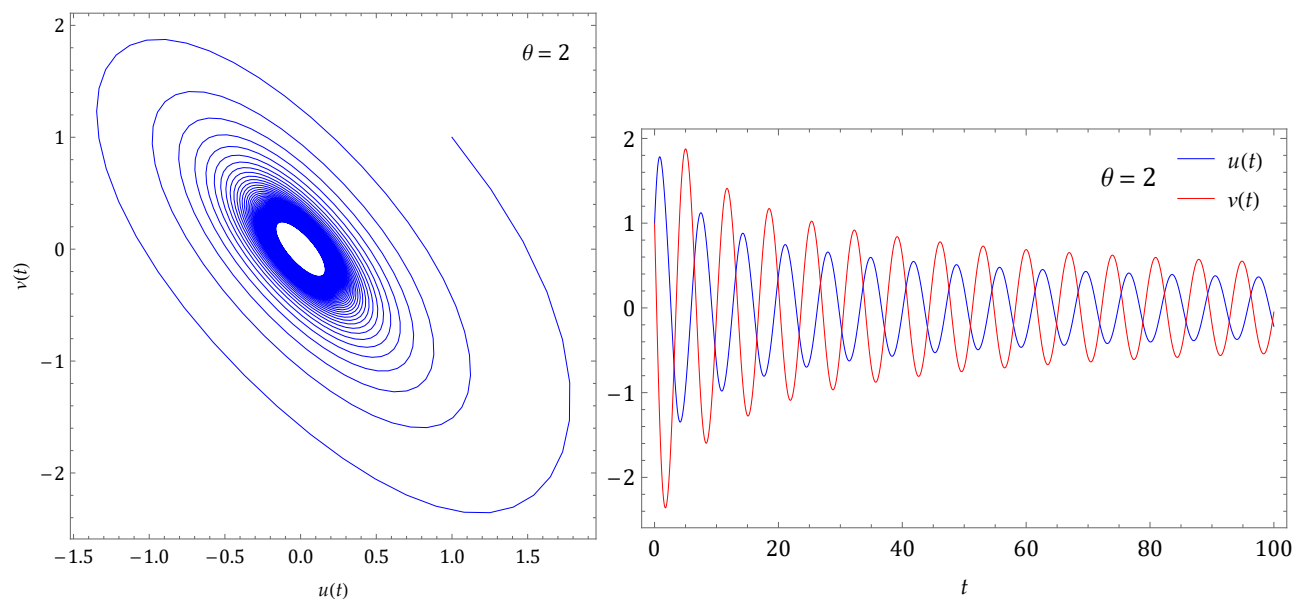

(a)
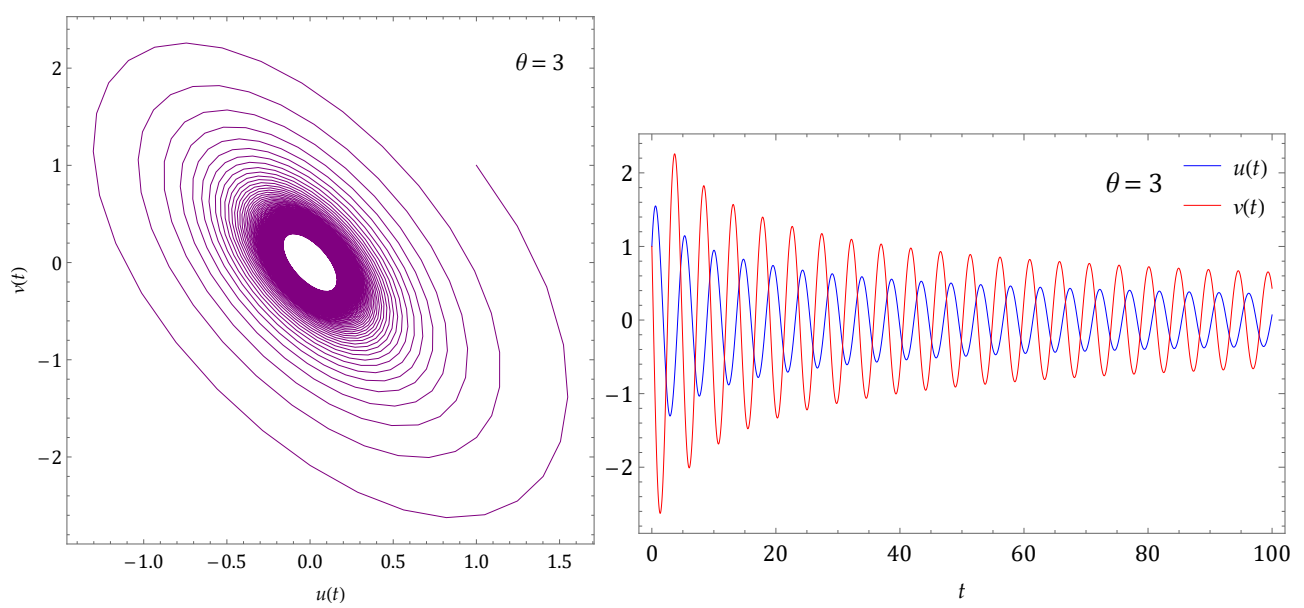

(b)
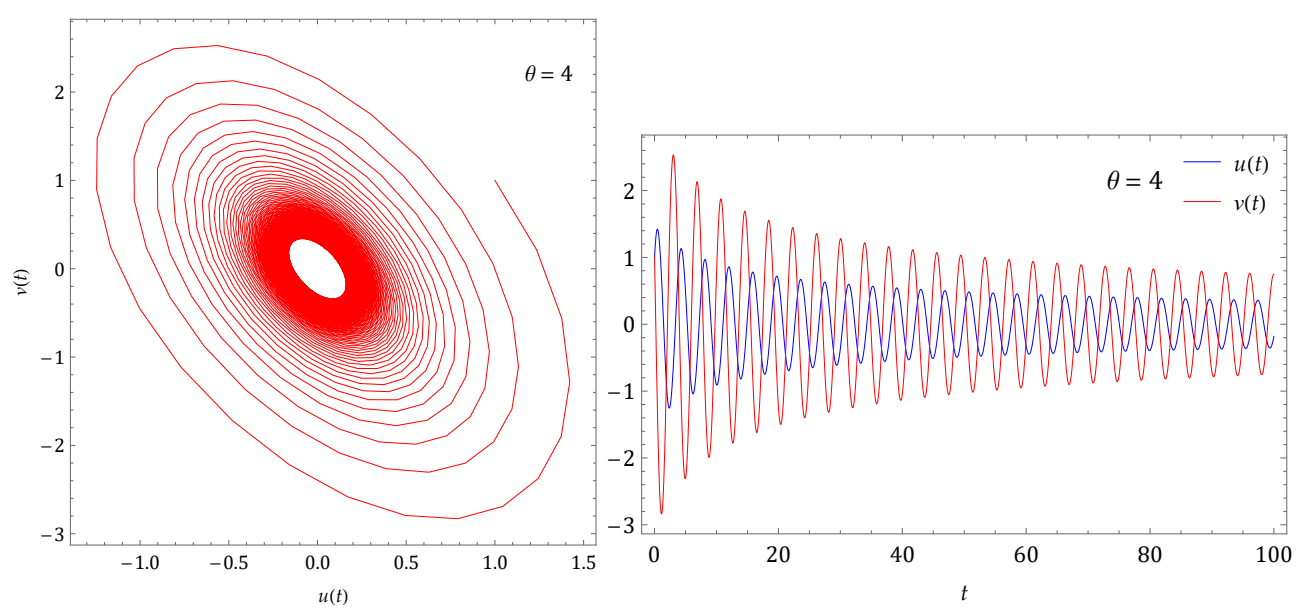

(c)

Figure 3. Response of the results achieved for $(a) \theta=2$, (b) $\theta=3$ and $(c) \theta=4$ at $\beta=1, \eta=1, \mu=0.95, \gamma=1$ and $\epsilon=0.1$.

\section{Conclusion}

In the present study, we analysed the atmospheric ocean model within the frame of the novel fractional operator using an efferent numerical scheme. The complex nature of the considered ENSO model captured for distinct fractional order in parametric plots. 
The behaviour for different parameters associated with coupled system is analysed and presented in plots. The conditions for both existence and uniqueness are archived in the present study for the considered system with the aid of Fixed-point theory and Banach space.

The capture plots show that the hired system is exceptionally reliant on the fractional operator. The projected method finds the solution for the employed system without making any perturbation, transformations, or discretization. The consequences attained in the present study are simulating as related to results available in the literature. Moreover, the hired system plays an important part in weather forecast and ocean consequences related to the daily life of the living beings. Hence, the present investigation can aid the researchers to investigate more regarding the model and opens the door for innovation. Finally, the efficiency and reliability of both considered operator and algorithm can be evidence with the help of the present investigation and further, they can employ for the complex model to study corresponding consequences.

\section{Declarations}

\section{Consent for publication}

Not applicable.

\section{Conflicts of interest}

The author declares that he has no conflict of interests.

\section{Funding}

Not applicable.

\section{Acknowledgements}

The author is thankful to the reviewers and editor for their valuable suggestions to improve the quality of the manuscript.

\section{References}

[1] M. Caputo, Elasticita e Dissipazione, Zanichelli, Bologna, (1969).

[2] K. S. Miller, B. Ross, An introduction to fractional calculus and fractional differential equations, A Wiley, New York, (1993).

[3] I. Podlubny, Fractional Differential Equations, Academic Press, New York, (1999).

[4] A. A. Kilbas, H. M. Srivastava, J. J. Trujillo, Theory and applications of fractional differential equations, Elsevier, Amsterdam, (2006).

[5] D. Baleanu, Z.B. Guvenc, J.A. Tenreiro Machado, New trends in nanotechnology and fractional calculus applications, Springer Dordrecht Heidelberg, London New York, (2010).

[6] M. Caputo, M. Fabrizio, A new definition of fractional derivative without singular kernel, Progr. Fract. Diff. Appl., 1 (2) (2015), 73-85.

[7] K. M. Safare, et.al., A mathematical analysis of ongoing outbreak COVID-19 in India through nonsingular derivative, Numerical Methods for Partial Differential Equations 37 (2) (2021), 1282-1298.

[8] M. Yavuz, European option pricing models described by fractional operators with classical and generalized Mittag-Leffler kernels, Numerical Methods for Partial Differential Equations, (2021), DOI: 10.1002/num.22645.

[9] L. Akinyemi, M. Şenol, S. N. Huseen, Modified homotopy methods for generalized fractional perturbed Zakharov-Kuznetsov equation in dusty plasma, Adv. Differ. Equ., 45 (2021), DOI: 10.1186/s13662-020-03208-5.

[10] C. Baishya, S. J. Achar, P. Veeresha, D. G. Prakasha, Dynamics of a fractional epidemiological model with disease infection in both the populations, Chaos, 31 (2021), DOI: 10.1063/5.0028905.

[11] J. Fei-Fei, An equatorial ocean recharge paradigm for ENSO. Part I: conceptual model, J. Atmos. Sci., 54 (7) (1996), 811-829.

[12] Y. Zen, The Laplace-Adomian-Pade technique for the ENSO model, Math. Probl. Eng, 4 (2013), DOI:10.1155/2013/954857.

[13] J. Q. Mo, W. T. Lin, J. Zhu, The variational iteration solving method for El Nino/La Nino-Southern Oscillation model, Adv. Math., 35 (2) (2006), 232-236.

[14] J. Q. Mo, W. T. Lin, Generalized variation iteration solution of an atmosphere-ocean oscillator model for global climate, J. Syst. Sci. Complex, 24 (2) (2011), 271-276.

[15] Z. Xian-Chun, L. Yi-Hua, W. T. Lin, J. Q. Mo, Homotopic mapping solution of an oscillator for the El nino/La Nina-Southern Oscillation, Chin. Phys. B, 18 (11) (2009), 4603-4605.

[16] J. Singh, D. Kumar, J. J. Nieto, Analysis of an El Nino-Southern Oscillation model with a new fractional derivative, Chaos Solitons Fractals, 99 (2017), 109-115.

[17] M. Gubes, H. A. Peker, G. Oturanc, Application of differential transform method for El Nino Southern Oscillation (ENSO) model with compared Adomian decomposition and variational iteration methods. J. Math. Comput. Sci., 15 (2015), 167-178.

[18] J. Q. Mo JQ, W. T. Lin, Perturbed solution for the ENSO nonlinear model, Acta Phys. Sinica., 53 (4) (2004), $996-998$.

[19] L. Akinyemi, O.S. Iyiola, A reliable technique to study nonlinear time-fractional coupled Korteweg-de Vries equations, Adv. Differ. Equ., 2020 (2020), 1-27, DOI: 10.1186/s13662-020-02625-w.

[20] E. K. Akgül, A. Akgül, M. Yavuz, New Illustrative Applications of Integral Transforms to Financial Models with Different Fractional Derivatives, Chaos Solitons Fractals 146 (2021), 110877. 
[21] P. Veeresha, E. Ilhan, H. M. Baskonus, Fractional approach for analysis of the model describing wind-influenced projectile motion, Phys. Scr., 96 (2021), DOI: 10.1088/1402-4896/abf868.

[22] L. Akinyemi, A fractional analysis of Noyes-Field model for the nonlinear Belousov-Zhabotinsky reaction, Comp. Appl. Math., 39 (2020), 1-34, DOI: 10.1007/s40314-020-01212-9.

[23] P. Veeresha, D. G. Prakasha, A reliable analytical technique for fractional Caudrey-Dodd-Gibbon equation with Mittag-Leffler kernel, Nonlinear Eng., 9 (1) (2020), 319-328.

[24] M. Yavuz, N. Sene, Fundamental calculus of the fractional derivative defined with Rabotnov exponential kernel and application to nonlinear dispersive wave model, J. Ocean Eng. Sci., 6 (2) (2021), 196-205.

[25] S.-W. Yao, E. Ilhan, P. Veeresha, H. M. Baskonus, A powerful iterative approach for quintic complex Ginzburg-Landau equation within the frame of fractional operator, Fractals, (2021), DOI: 10.1142/S0218348X21400235.

[26] L. Akinyemi, P. Veeresha, M. Senol, Numerical solutions for coupled nonlinear Schrodinger-Korteweg-de Vries and Maccari's systems of equations, Modern Physics Letters B, (2021), 2150339, DOI: 10.1142/S0217984921503395.

[27] A. Atangana, R. T. Alqahtani, Numerical approximation of the space-time Caputo-Fabrizio fractional derivative and application to groundwater pollution equation, Adv. Diff. Equ., 1 (2016), 1-13.

[28] C. Baishya, Dynamics of a Fractional Stage Structured Predator-Prey model with Prey Refuge, Indian J. Ecol., 47 (4) (2020), $1118-1124$.

[29] P. Veeresha, D.G. Prakasha, H.M. Baskonus, New numerical surfaces to the mathematical model of cancer chemotherapy effect in Caputo fractional derivatives, Chaos 29 (013119) (2019). DOI: 10.1063/1.5074099.

[30] K. M. Owolabi, A. Atangana, Analysis and application of new fractional Adams-Bashforth scheme with Caputo-Fabrizio derivative, Chaos, Solitons Fractals, 105 (2017), 111-119.

[31] A. Atangana, Derivative with a new parameter: theory, methods and applications, New York: Academic Press; 2016.

[32] A. Atangana, K. M. Owolabi, New numerical approach for fractional differential equations, Math. Model. Nat. Phenom., 13 (2018), DOI: $10.1051 / \mathrm{mmnp} / 2018010$.

[33] A. Atangana, J. J. Nieto, Numerical solution for the model of RLC circuit via the fractional derivative without singular kernel, Adv. Mech. Eng., 7(2015), 1-6.

[34] M. Caputo, M. Fabrizio, Applications of new time and spatial fractional derivatives with exponential kernels, Progr. Fract. Diff. Appl., 2 (2016), 1-11.

[35] J. Losada, J. J. Nieto, Properties of the new fractional derivative without singular kernel, Progr. Fract. Differ. Appl., 1 (2015), 87-92.

[36] J. Danane, K. Allali, Z. Hammouch, Mathematical analysis of a fractional differential model of HBV infection with antibody immune response, Chaos Solitons Fractals, 136 (2020).

[37] K. M. Owolabi, Z. Hammouch, Spatiotemporal patterns in the Belousov-Zhabotinskii reaction systems with Atangana-Baleanu fractional order derivative, Phys. A, 523 (2019), 1072-1090.

[38] F. Haq, I. Mahariq, T. Abdeljawad, N. Maliki, A new approach for the qualitative study of vector born disease using Caputo-Fabrizio derivative, Numer. Methods Partial Differ. Equ., 37 (2) (2021), 1809-1818.

[39] P. Veeresha, H. M. Baskonus, W. Gao, Strong interacting internal waves in rotating ocean: Novel fractional approach, Axioms, 10 (2) (2021).

[40] W. Zhong, L. Wang, T. Abdeljawad, Separation and stability of solutions to nonlinear systems involving Caputo-Fabrizio derivatives, Adv. Differ. Equ., 166 (2020). DOI: 10.1186/s13662-020-02632-x.

[41] R. Gul, M. Sarwar, K. Shah, T. Abdeljawad, F. Jarad, Qualitative Analysis of Implicit Dirichlet Boundary Value Problem for Caputo-Fabrizio Fractional Differential Equations, J. Funct. Spaces, (2020), DOI: 10.1155/2020/4714032.

[42] M. Yavuz, E. Bonyah, New approaches to the fractional dynamics of schistosomiasis disease model, Phys. A, 525 (2019), 373-393.

[43] P. Veeresha, D. G. Prakasha, Z. Hammouch, An efficient approach for the model of thrombin receptor activation mechanism with Mittag-Leffler function, Nonlinear Analysis: Problems, Applications and Computational Methods, (2020), 44-60.

[44] K. Shah, F. Jarad, T. Abdeljawad, On a nonlinear fractional order model of dengue fever disease under Caputo-Fabrizio derivative, Alexandria Eng. J., 59 (4) (2020), 2305-2313.

[45] M. Yavuz, N. Sene, Fundamental calculus of the fractional derivative defined with Rabotnov exponential kernel and application to nonlinear dispersive wave model, J. Ocean Eng. Sci., 6 (2) (2021), 196-205.

Mathematical Modelling and Numerical Simulation with Applications (MMNSA) (http://www.mmnsa.org)

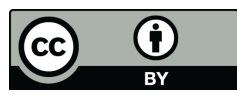

Copyright: $(2) 2021$ by the authors. This work is licensed under a Creative Commons Attribution 4.0 (CC BY) International License. The authors retain ownership of the copyright for their article, but they allow anyone to download, reuse, reprint, modify, distribute, and/or copy articles in MMNSA, so long as the original authors and source are credited. To see the complete license contents, please visit (http://creativecommons.org/licenses/by/4.0/). 